TUBERCULOUS PERICARDITIS

\title{
Tuberculous pericarditis: Challenges and controversies in the modern era
}

Charles G. Kyriakakis

Division of Cardiology, Department of Medicine, University of Stellenbosch and Tygerberg Academic Hospital, South Africa.

Address for correspondence:

Charles G. Kyriakakis

Division of Cardiology, Department of Medicine

University of Stellenbosch and Tygerberg Hospital

PO Box 19063

Tygerberg

7505

South Africa

Email:

cgk@sun.ac.za

\section{INTRODUCTION}

Mycobacterium tuberculosis (MTB) has been ravaging mankind through the ages with the earliest evidence of infection dating back to the Neolithic era, 9000 years ago, confirmed by the extraction of MTB DNA from archeological human remains. ${ }^{(2)}$ It is considered to be the most lethal pathogenic killer in human history, with the developing world experiencing 90\% of cases. ${ }^{(1)}$ South Africa (SA) continues to have the highest incidence and prevalence of TB in the world and this places an immense burden on the health of our population. (I) The developed world has enjoyed a low incidence of the disease, with new cases predominantly identified in foreign-borne residents and immigrants from developing nations. However, an inevitable resurgence in infection rates threatens Europe, given the recent explosion in the number of refugees fleeing to that continent. In SSA the high rate of co-infection with the Human Immunodeficiency Virus (HIV) is associated with increased mortality. In 2013 the prevalence of HIV in TB incident cases in SA was $61 \%$, second only to Zimbabwe at $72 \%$. (1) Despite the global health threat posed by this age-old disease, which is responsible for more deaths worldwide than any other cause, there has been little in the way of new drug development. The standard short-course therapeutic regime has been in use for over 30 years. ${ }^{(3)}$ The emergence of multidrug resistant (MDR), extensive drug resistant (XDR) and total drug resistant (TDR) strains poses a significant threat, particularly in SA where the rate of MDR infection stands at $1.8 \%$ of all new cases and $6.7 \%$ of retreatment cases. ${ }^{(4)} \mathrm{SA}$ also has the highest number of XDR cases in the world with $10 \%$ of the MDR group being

\section{ABSTRACT}

Tuberculous pericarditis (TBP) continues to wreak havoc across Sub-Saharan Africa (SSA). Despite more than 5 decades of treatment and research into TBP, we are not much closer to alleviating the suffering and mortality associated with this extrapulmonary manifestation of tuberculosis (TB). In the era of modern cardiology, diseases of the pericardium do not receive the same amount of research attention and investment as what diseases of lifestyle do. Interventional techniques for their diagnosis and management do not extend much further than pericardiocentesis with appropriate laboratory investigations. They also do not provide the potential for the development and use of consumable equipment, or that of expensive drugs and, consequently, financial investment into their research and development is not forthcoming. Diseases of the pericardium do, however, remain important within the discipline that we practice and TBP in particular deserves our continuous efforts and attention. It is unfortunate to acknowledge that we have not made much of an impact on this ancient foe over the last $\mathbf{5 0}$ odd years. Despite the World Health Organisation (WHO) declaring TB a global emergency in 1993, more than 20 years later we are not much better off. In 2013 an estimated 9 million people developed the disease and 1.5 million died from it.(1) TBP is predominantly a disease of SSA and it requires a solution from the very region which it torments. SAHeart 2016;13:104-1 I I

$X D R .^{(4)}$ Extra-pulmonary infections account for $10-42 \%$ of TB cases with TBP occurring in I - $2 \%$ of patients with pulmonary TB. ${ }^{(5,6)}$ In SA, TB accounts for 50 - 70\% of large pericardial effusions in HIV negative patients and $>90 \%$ in those who are HIV positive. (7) The morbidity and mortality remain high despite anti-TB chemotherapy with a case fatality rate of $26 \%$ at 6 months, rising to $40 \%$ in patients with HIV co-infection. ${ }^{(8)}$ Rifampicin based anti-tuberculous therapeutic regimens decreased the risk of constrictive pericarditis (CP), the leading cause of mortality, from $50 \%$ to 14 - 40\%. ${ }^{(9)}$ This remains an unacceptably high case rate. To date, no adjunctive therapy has been proven to alter the rate of death from constriction in TBP. (10-13) Tuberculous meningitis, another extrapulmonary manifestation of the disease, which is also responsible for considerable morbidity and mortality, has recently been the target of an attempt at an intensified 
therapeutic regimen. In adults with the disease in Vietnam, an increased dose of rifampicin, and the addition of levofloxacin to the standard regimen, disappointingly did not reduce the overall mortality that stands at $28 \%$. ${ }^{(14)}$ In TBP, extending the short-course treatment regimen from 6 - 9 months, does not alter outcomes. ${ }^{(15)}$

Regardless of the global health threat posed by TB, inadequate funding into TB research and development (R\&D) in 2014 left a funding gap of $\$ 1.3$ billion measured against the $\$ 2$ billion annual investment required for by the Global Plan to Stop TB 2011 - 2015.(16) Despite the shortfall in TB R\&D funding over the past 5 years, strides have been made into the development of new drugs against drug-resistant $\mathrm{TB}$, a more rapid diagnostic test (validated for use in pulmonary TB) and to re-invigorate the once-dormant field of TB vaccine research. Bedaquiline, the first drug in over 40 years to have a new mechanism of action against TB, inhibits mycobacterial ATP synthase. ${ }^{(17)}$ In 2012 it received accelerated FDA approval for use in MDR pulmonary TB. Although there have been recent advances in TBP, controversies and challenges still exist and this article will outline specific areas where attention should be focused.

\section{ESTABLISHING THE DIAGNOSIS}

In areas with a high prevalence of TBP, empiric therapy is recommended when other potential causes have been excluded. Excluding other potential aetiologies of pericarditis is not always a simple undertaking, and erroneously exposing patients without a diagnosis of TBP to antituberculous chemotherapy is not without risk. In regions where clinicians do not have the ability to undertake pericardiocentesis, or where pericardial fluid collections are too small for safe needle aspiration, a clinical scoring system can be used to establish a probable diagnosis with a sensitivity and specificity of $86 \%$ and 85\% respectively (Table I). ${ }^{(18)}$ Establishing a confirmed diagnosis remains challenging as TB culture of pericardial fluid is slow and insensitive and direct examination for acid fast bacilli (AFBs) has a low yield. ${ }^{(19)}$ Clinicians therefore rely on diagnostic algorithms, incorporating biochemical markers such as adenosine deaminase (ADA) and unstimulated interferon gamma (uIFN- $\gamma$ ), to make a presumptive diagnosis of TBP. ${ }^{(20)}$ Whilst an ADA level

TABLE I: The Tygerberg Diagnostic Index Score for TBP. A score $\geq 6$ has a sensitivity of $86 \%$ and a specificity of $85 \%$ for the diagnosis of TBP.

\begin{tabular}{lc}
\hline \multicolumn{1}{c}{ Criterion } & Score \\
\hline Weight loss $(>10 \%)$ & 1 \\
\hline Night sweats & 1 \\
\hline Fever $>37.2^{\circ}$ Celsius & 2 \\
\hline Serum globulin $>40 \mathrm{~g} / 1$ & 3 \\
\hline White cell count $<10 \times 10^{\circ} / 1$ & 3 \\
\hline
\end{tabular}

$\geq 40 \mathrm{IU} / \mathrm{L}$ in the setting of an exudative effusion with a lymphocyte: neutrophil ratio $>$ I has a high sensitivity and specificity for the disease, it does not come without its challenges. ${ }^{(18,20)}$ An accurate determination of ADA is frequently not possible, particularly in the setting of blood stained effusions. A pericardial fluid uIFN- $\gamma$ level $>50 \mathrm{pg} / \mathrm{ml}$ is both more sensitive, and specific, than ADA for the diagnosis of TBP. ${ }^{(18,20)}$ It is, however, not widely available as a diagnostic test in SSA due to its high cost and the fact that it requires expertise on the part of the laboratory performing the test to deliver a reliable result. To date its application has been predominantly in a research setting. Xpert-MTB/RIF, an automated nucleic acid amplification test, which can rapidly detect both MTB DNA (within 2 hours) and assess for possible Rifampicin resistance, has been approved for sputum evaluation in the setting of possible pulmonary TB. It has been demonstrated to have a low sensitivity in the setting of pleural TB, $46 \%$ vs. $99 \%$ by culture, and more recently its potential value was evaluated in the setting of pericardial disease on pericardial fluid specimens. ${ }^{(21,22)}$ It achieved a low sensitivity of $63,8 \%$ and was found to be inferior to both ADA and $\mathrm{uIFN}-\boldsymbol{\gamma}$ in microbiologically confirmed TBP. (22) Its potential value in the evaluation of pericardial tissue specimens remains unknown. An older PCR assay was evaluated in 20 patients, who underwent open surgical pericardial biopsy, on both fluid and tissue samples. ${ }^{(23)}$ The sensitivity of PCR was higher for tissue specimens (80\%) than fluid specimens (15\%) and we are therefore evaluating the potential diagnostic accuracy of the newer Xpert-MTB/RIF assay on both pericardial fluid and tissue specimens obtained via a non-surgical percutaneous approach.

Until recently, access to pericardial tissue required invasive surgical techniques known to both prolong hospital stay and increase patient morbidity and mortality. Percutaneous pericardioscopy is a minimally invasive technique with a low complication rate for obtaining pericardial tissue, allowing for a more detailed evaluation of the diseased pericardium than evaluation of fluid specimens alone. ${ }^{(24)}$ Through the introduction of a flexible fibre-optic scope into the pericardial space, both the epicardium and parietal pericardium can be assessed for areas of inflammation, allowing targeted biopsies to be acquired under direct vision (Figure I). It has been demonstrated to have a low risk profile in large series, with not a single reported mortality attributable to the procedure. ${ }^{(24-28)}$ The risk of the procedure is the inherent risk associated with the pericardiocentesis procedure itself, and not pericardioscopy. In the Marburg registry, including 259 patients, pericardial biopsy by this technique allowed for a more definitive aetiological classification and targeted therapy in patients presenting with pericardial effusion in a first world setting.(26) The investigators in this registry successfully used a 6-French endomyocardial bioptome to acquire suitably representative tissue samples without an increase in procedural related adverse events. It has 


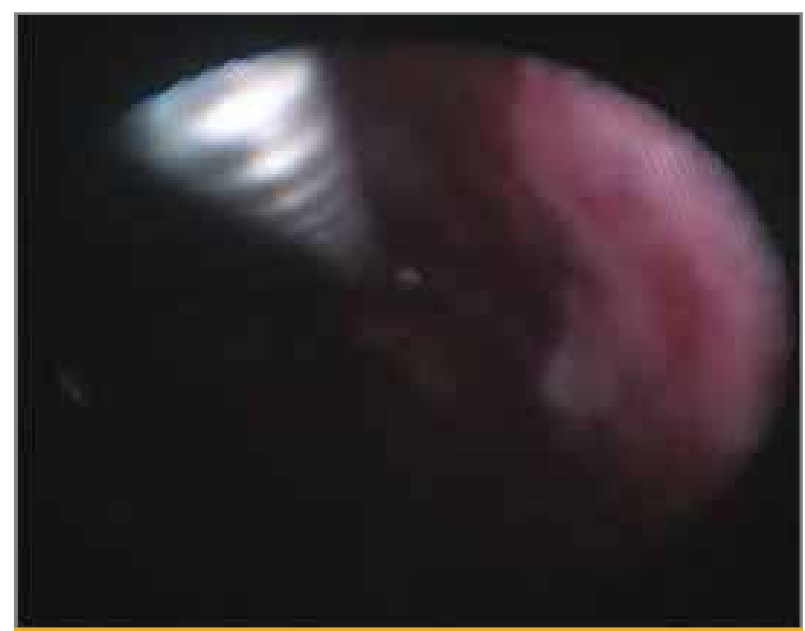

FIGURE I and accompanying online video: Intrapericardial view during percutaneous pericardioscopy with targeted epicardial biopsy. Biopsy forceps visible at II o'clock.

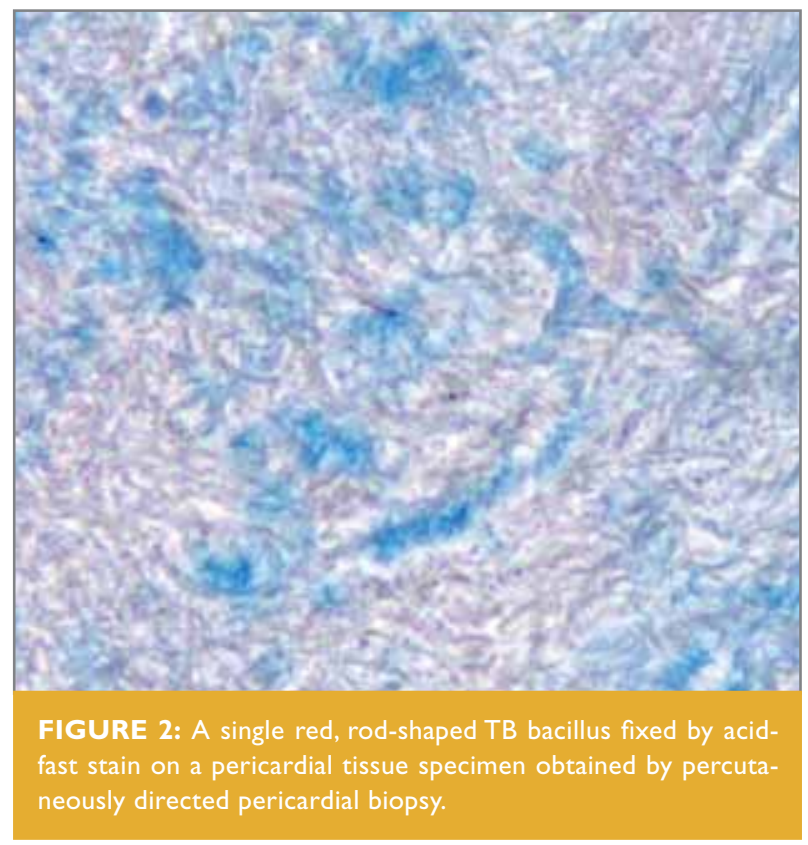

also been proven that extensive sampling (18 - 20 biopsies) improves the diagnostic yield of the procedure, whilst not impacting on procedural safety as compared to limited sampling of 4 - 6 biopsies. ${ }^{(24)}$ The probability of diagnosing an infiltrate is also significantly higher in epicardial than in parietal pericardial sampling ( $80 \%$ vs. $7 \%$ in neoplastic disease, and $93 \%$ vs. $14 \%$ in inflammatory disease). ${ }^{(29)}$ Preferential epicardial sampling, directly from the surface of the beating heart, does not adversely influence procedural safety. ${ }^{(29)}$

In our pilot study, which included 3 I patients, we confirmed that it is a minimally invasive procedure with low procedural risk when applied to a population with a high prevalence of TBP. ${ }^{(30)}$ By means of limited sampling of 6 biopsies, using a I mm bioptome, representative samples could safely be acquired in 27 participants. A diagnosis of definite TBP was established in $55 \%$, all of which were diagnosed on tissue specimens, whilst fluid evaluation missed the diagnosis in 20\%. A rapid diagnosis could be achieved in $73 \%$ by the demonstration of AFBs on tissue samples, allowing clinicians to confidently institute antiTB chemotherapy (Figure 2). All fluid samples were AFB negative, and a fluid based diagnosis was reliant on TB culture, with a mean time to positive culture of over three weeks. An alternative diagnosis was established in 17\%, who could be spared unnecessary TB chemotherapy. A single case of MDRTB was identified in this cohort, again diagnosed on a tissue specimen and missed on TB culture of pericardial fluid. ${ }^{(30)}$ Establishing a definite diagnosis of TBP, and allowing for drugsensitivity testing, is of paramount importance now that we have entered the era of MDR, XDR and TDR strains of the disease. It is estimated that only 10\% of MDR TB cases are diagnosed worldwide, and only half of them receive appropriate treatment. ${ }^{(3 I)}$

Percutaneous pericardioscopy remains a research tool, and it is unlikely that its use will disseminate farther afield than specialised pericardial disease research centers due to the infrastructure required to perform the technique. It does, however, offer the opportunity to safely acquire pericardial tissue specimens and has been demonstrated to potentially offer superior diagnostic accuracy over the evaluation of fluid samples alone in a population with a high prevalence of TBP. This can be done with a limited sampling technique and comparatively smaller sampling forceps compared to studies performed in the developed world. ${ }^{(30)}$ No reliable data is available on mycobacterial speciation, strain typing, and the incidence of drug resistant disease or mycobacterial tropism in the setting of TBP in a population with, and without, HIV coinfection. A recent study, undertaken at our institution, assessing the effect of mycobacterial genotype and its association on disease phenotype in a paediatric population, included only one single patient with pericardial disease out of a cohort of 392 subjects. ${ }^{(32)}$ Pericardial involvement is far less common in the paediatric population and thus assessment in the adult setting is required. Apart from an impaired host immune response favouring the development of extrapulmonary disease, the authors hypothesised that different MTB strains, in particular the Beijing genotype, could cause distinct clinical disease phenotypes, as suggested by animal studies in which different MTB strains evoke markedly different immunopathological host responses. Extrapulmonary strains have previously been demonstrated to infect macrophages more efficiently whilst also being more infectious than pulmonary strains, a feature suggesting greater pathogenicity. ${ }^{(33)}$ The Beijing genotype, which 
predominates in South Africa, is well known to increase the risk of central nervous system involvement and more severe pulmonary disease. Whether or not this is the case for pericardial disease remains to be defined in a study by our research group. In the report on paediatric disease, the Beijing genotype was associated more commonly with extra-thoracic disease than the LAM genotype, suggesting that LAM strains have less ability to disseminate. ${ }^{(32)}$ No evidence of mixed MTB genotype infection was demonstrated in any of the participants of this paediatric cohort.

Whilst rates of drug resistance in pulmonary disease are well characterised, the incidence in pericardial involvement is currently unknown. A study conducted in KwaZulu-Natal has revealed that S/Quebec strains (34\%) were more commonly associated with MDR disease and LAM strains (89\%) with XDR-TB, whilst Beijing strains were predominantly responsible for drug susceptible disease. ${ }^{(34)}$ Although they evaluated 297 isolates from 227 patients with TB, with almost 25\% having extra-pulmonary disease, the authors did not identify the various sites of extrapulmonary involvement and the incidence for pericardial disease remains unknown.

An accurate point of care test for establishing the diagnosis of TBP does not exist. We are currently exploring the potential of developing such a test by identifying a biosignature of a combination of cytokines. Pericardioscopy offers the opportunity to accurately validate such a combination of cytokines against a confirmed histological and microbiological diagnosis of MTB pericarditis, as provided by pericardial tissue specimens. It is possible that a higher yield of MTB can be gained with the acquisition of larger tissue fragments. A pilot trial is planned to evaluate the safety of employing a larger pericardioscope that will accommodate a 6-French bioptome in our patient population.

\section{THE CONTROVERSIAL ROLE OF STEROIDS}

Attempts at modulating the host inflammatory response to TBP with glucocorticoids, in an attempt to prevent constriction, reach as far back as 1959 when 14 patients received both TB therapy and adjunctive high-dose steroids, whilst another 14 received TB therapy alone. There was no difference in the development of $\mathrm{CP}$ in either of the two groups. ${ }^{(10)} \mathrm{A}$ systematic review of randomised controlled trials, evaluating the potential advantage of adjunctive corticosteroids, found no significant beneficial effect of steroids on either the re-accumulation of pericardial effusion or the progression to CP.(35)

In our local experience complete percutaneous drainage of a tuberculous effusion, in combination with anti-tuberculous therapy, is associated with an extremely low risk for constriction and cardiac death. ${ }^{(9,11)}$ One small trial has shown that the addi- tion of intrapericardial urokinase may facilitate this approach. ${ }^{(12)}$ Furthermore, in an underpowered trial of 57 patients, the administration of intrapericardial steroids did not influence the risk of developing constriction. (1) Patients with effusive constrictive pericarditis (ECP) were excluded from enrolment and those with purely effusive disease were randomised to 1 of 3 arms. These included antituberculous chemotherapy for 6 months with placebo, oral prednisolone or intrapericardial triamcinolone. All participants underwent complete pericardial drainage with intermittent daily aspiration until $<100 \mathrm{mls} / 24 \mathrm{hr}$ had been drained. At one year's follow up there was only I case of $\mathrm{CP}$, having occurred in a patient randomised to oral prednisone. The authors concluded that short course antituberculous chemotherapy, in combination with extended pericardial drainage, results in excellent outcomes in patients with effusive TBP. More recently, the IMPI trial evaluated the value of adding adjunctive high-dose oral prednisolone for 6 weeks to anti-TB therapy in both $\mathrm{HIV}+$ and - patients with TBP. (13) Two thirds of the I 400 participants underwent pericardiocentesis and a definite diagnosis of TBP was established in approximately 16\%. Adjunctive oral prednisolone had a neutral effect on the combined primary outcome of death from all causes, cardiac tamponade requiring pericardiocentesis and pericardial constriction. In the HIV positive group it was associated with an adverse outcome in that it led to an increase in HIV-related malignancies and a trend towards HIVrelated death. The rate of constriction, a secondary outcome, was reduced by $46 \%$ regardless of HIV status without translating into a reduction in mortality. This is a difficult finding to explain given that it is the very development of constriction that is responsible for the late mortality seen in TBP. The 2015 European Society of Cardiology (ESC) guidelines for the diagnosis and management of pericardial diseases has therefore allocated a class $\mathrm{ll}$ b, level of evidence $\mathrm{C}$ recommendation to the addition of adjunctive corticosteroids in HIV negative patients with TBP. ${ }^{(36)}$ They should clearly be avoided in patients who are HIV positive. The recommendations on adjunctive oral steroid therapy still need to be updated in the guidelines issued by the American Thoracic Society, Centers for Disease Control and Infectious Diseases Society of America, in which they are recommended in all cases of TBP, regardless of HIV status. ${ }^{(37)}$ The IMPI trial protocol relied heavily on clinical findings supportive of constriction, and did not incorporate the use of mitral annular Tissue Doppler Imaging (TDI) in the transthoracic echocardiographic (TTE) criteria for establishing the diagnosis of constriction. ${ }^{(38)}$ Radial and circumferential ventricular expansion are limited in constriction as the myocardium is tethered to the pericardium, however, the subendocardial longitudinal fibers are free to lengthen and thus early annular diastolic tissue velocity $\left(\mathrm{e}^{\mathrm{I}}\right)$ is correspondingly normal or supra-normal. ${ }^{(39)}$ This is a paradoxical finding in the setting of elevated left atrial filling pressures and the $E / \mathrm{e}^{\mathrm{I}}$ ratio is therefore low, despite an ele- 
vated left ventricular end-diastolic pressure, producing the TTE feature of annular paradox. ${ }^{(40)}$ Understandably, given that the IMPI trial was conducted across the continent where resources and expertise are limited, invasive haemodynamics were not employed to confirm a suspected diagnosis of CP. This is in line with standard practice, provided an accurate diagnosis of constriction can be confirmed by detailed TTE evaluation incorporating mitral annular TDI.(36) More recently, respiratophasic septal shift, in combination with either a hepatic venous expiratory diastolic flow reversal ratio $\geq 0.79$, or a medial annular TDI $\mathrm{e}^{\mathrm{I}} \geq 9 \mathrm{~cm} / \mathrm{sec}$ has been demonstrated to be $87 \%$ sensitive and 91\% specific for CP. ${ }^{(4)}$ It is therefore possible that subclinical cases of constriction went undiagnosed in this patient cohort. This offers a potential explanation for why a perceived reduction in constriction failed to translate into a derived mortality benefit in the group receiving adjunctive corticosteroid therapy.

In the modern era, a detailed TTE, including: annular TDI and hepatic venous Pulsed-wave (PW) Doppler (Figure 3) should be undertaken in all patients with suspected CP. ${ }^{(41)}$ Annular TDI assessment has the added advantage of identifying restrictive cardiomyopathy as a cause for elevated left atrial filling pressures. Here the medial mitral annular early diastolic e velocity will be $<8 \mathrm{~cm} / \mathrm{sec}$ due to underlying primary myocardial disease. ${ }^{(40,42)}$

It is unlikely that all HIV negative patients with TBP stand to derive benefit from the addition of high-dose adjunctive corti-

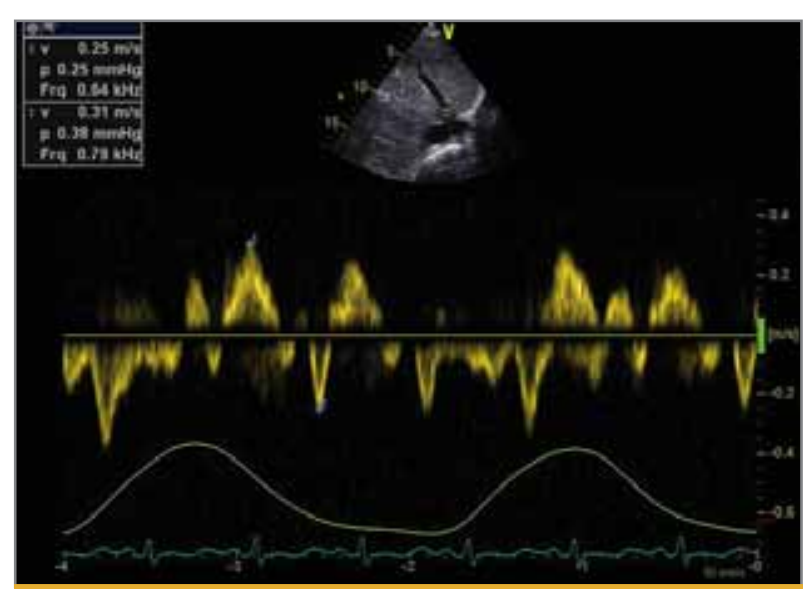

FIGURE 3: Hepatic venous PW-Doppler assessment in a patient with CP. The hepatic venous expiratory early diastolic reversal ratio is defined as the expiratory diastolic reversal velocity divided by the diastolic forward velocity. In this example the ratio is I.24. It is a reflection of the two hallmark physiological features of CP: the dissociation of intrathoracic and intracardiac pressures and enhanced interventricular dependence. Relatively faster left-sided cardiac chamber filling during expiration causes respiratophasic ventricular septal shift into the right ventricle. This reduces the hepatic vein forward velocity and exaggerates the late diastolic reversal velocity. costeroids as the majority will not go on to constrict, provided they undergo complete pericardial drainage and remain compliant on their antituberculous therapy. It would therefore be prudent to identify the group which is at highest risk of going on to develop CP as they stand to derive maximum benefit from adjunctive steroid therapy. Such a group remains undefined and clarity is required in this regard. It is our opinion that it is the group presenting with effusive-constrictive physiology that stands to benefit most and we are currently exploring this option. Effusive-constrictive pericarditis (ECP), which is due to visceral pericardial restriction, is traditionally a diagnosis established by invasive haemodynamics, requiring that right atrial (RA) pressure remains above $10 \mathrm{mmHg}$ or does not fall by $50 \%$ following complete pericardial fluid drainage with the normalisation of intrapericardial pressure. ${ }^{(43)}$ Other potential causes for a persistently elevated right atrial pressure, after the normalisation of intrapericardial pressure post pericardiocentesis do however exist, and recent data from our group demonstrates this fact. The finding of an elevated RA pressure does therefore not necessarily equate to constrictive physiology without proving the two hallmark physiological features of CP, namely the dissociation of intrathoracic and intracardiac pressures and enhanced interventricular dependence. Our group has recently demonstrated that ECP can be accurately defined by detailed TTE evaluation, including TDI, before and after pericardiocentesis (under review for publication). Furthermore, this study revealed that in half of those who would be classified as having ECP by traditional criteria, the hallmark (and most sensitive and specific) physiological sign of CP on invasive haemodynamics, i.e. systolic discordance of left ventricular and right ventricular pressures, was absent. In this cohort TTE criteria for establishing a diagnosis of ECP correlated very well with the invasive demonstration of the dissociation of intrathoracic and intracardiac pressures, and also that of enhanced interventricular dependence, and not with elevated RA pressure after the normalisation of intrapericardial pressure. This would suggest that the traditional criteria for diagnosing ECP are over sensitive and outdated. With the availability of this new data an assessment of this particular syndrome of TBP can now be undertaken without the need for invasive, and potentially more hazardous, right heart catheterisation for research purposes. It is our hypothesis that it is this group who will potentially derive maximum benefit from the addition of high-dose adjunctive oral corticosteroid therapy in preventing the development of constriction, and ultimately the mortality associated with this pericardial syndrome.

\section{CONSTRICTION: SURGICAL TIMING, APPROACH,AND THE RISK OF RECURRENCE}

$\mathrm{CP}$ is a potentially curable cause of diastolic heart failure, generally developing within 6-months of presentation in the effusive stage. ${ }^{(44)}$ The incidence of tuberculous CP is 31,65 cases 
per 1000 person years, second only to purulent disease at 52,74 cases per I 000 person years. ${ }^{(45)}$ The 2015 ESC guidelines for the diagnosis and management of pericardial diseases provides a Class IC recommendation only for anti-TB therapy as a means to prevent constriction, whilst both adjunctive steroids (in HIV negative patients), and intrapericardial urokinase each receive a Class Ilb, C recommendation. ${ }^{(36)}$ Approximately $60 \%$ of patients with early tuberculous constriction will respond to antituberculous therapy. ${ }^{(46)}$ Pericardiectomy is recommended if after $4-8$ weeks of therapy the patient's condition remains unchanged, or deteriorates. ${ }^{(36)}$ This remains controversial due to limited data, however the general consensus is that resection is associated with lower surgical morbidity and mortality earlier in the course of pericardial adhesion, and before the onset of myocardial atrophy. No recommendation is provided for the preferred surgical access site or completeness of pericardial resection in TBP-CP within the guideline document. The choice of surgical approach, i.e. median sternotomy vs. left anterolateral thoracotomy is currently not definitive and is thus determined by the surgeon's preference. ${ }^{(47)}$ Anecdotal reports have indicated good operative results associated with the surgical technique used.(47,48,50) In a series from 1982, including 109 patients, 31 with histologically proven TB and the rest with probable TB, all underwent pericardiectomy via a left anterolateral thoracotomy. ${ }^{(48)} \mathrm{Via}$ this approach the pericardium could be mobilised anteriorly between both phrenic nerves, extended to the right ventricular outflow tract and also posteriorly and inferiorly over the diaphragmatic surface of the heart (radical pericardiectomy). The in hospital mortality rate in this series was just 3\% and only I case required a revision pericardiectomy at one year for recurrent constriction. The author reported a suspected low rate of recurrence. This was however judged solely on the basis of the clinical signs of a suspected recurrence of CP and TTE imaging was not employed. Furthermore, many patients did not attend follow up appointments and the low rate of recurrence was attributed to the absence of requests for disability or death certification. The study was also conducted before the era of HIV infection and the results are not necessarily applicable to our current practice. Interestingly, the potential advantage of this approach over that of a median sternotomy in offering access to wide pericardial resection, was more recently evaluated with 16 en bloc cadaver specimens and the review of surgical notes of both techniques. ${ }^{(49)}$ The investigators included a review of the surgical records from the series published in 1982 as part of the analysis. Although the anterior pericardium between phrenic nerves was found to constitute $58 \%$ of total selected pericardial area, only $26 \%$ of the total pericardium over the left ventricle was accessible from that approach. A left anterolateral approach was found to offer wider access to the left ventricle and $37 \%$ of total pericardial area could be accessed. This information therefore offers a functional anatomical perspective for the choice of left anterolateral thoracotomy as a surgical approach for pericardiectomy. In a retrospective report conducted during the HIV era, including 121 patients who underwent median sternotomy for $\mathrm{CP}$, the 30-day perioperative mortality rate was excessively high at $14 \% .{ }^{(50)}$ In other series reported worldwide this rate stands at $6-10 \%^{(51)}$. Only I $1.6 \%$ were HIV positive with $29.8 \%$ of cases having confirmed TBP with a further $61.2 \%$ having presumed TBP as the cause of their constriction. A preoperative low serum- $\mathrm{Na}+$ and/or $\mathrm{New}$ York Heart Association (NYHA) class IV effort tolerance were found to be independent predictors of early mortality. There were no early deaths in the HIV positive group, albeit this did make up a small percentage of the patient group. Sixty six percent of participants had a preoperative NYHA class III or $\mathrm{IV}$ effort tolerance, and it is in these groups where most of the 30 day mortality occurred, making up I I\% of the total $14 \%$ of those who succumbed. This is a likely indicator of end-stage disease complicated by myocardial atrophy. It is indeed well established that the group with a low cardiac index of $\leq 1.2 \mathrm{~L} / \mathrm{m}^{2} / \mathrm{min}$ at rest derive little or no benefit from surgery as surgical risk outweighs the potential benefit of symptomatic improvement. ${ }^{(52)}$ The investigators did not report on the rate of recurrent pericardial constriction, which to date remains undefined in the group with HIV co-infection undergoing pericardiectomy via median sternotomy. In a smaller cohort of 47 patients (46.8\% due to TBP) from Turkey, followed out to $61.2 \pm 66$ months, the rate of recurrent CP was zero. ${ }^{(53)}$ All but one patient underwent pericardiectomy via median sternotomy with radical pericardial resection. HIV status was not reported on and most patients had a preoperative NYHA class of II or III. The 30 day mortality rate was correspondingly low at $8.5 \%$.

In the developed world, where previous surgical pericardiotomy and prior chest radiotherapy are the most common aetiologies of $\mathrm{CP}$, repeat pericardiectomy is associated with a perioperative mortality rate of $20 \%$ at 30 days. ${ }^{(54)}$ The most common cause for this recurrence of constriction was found to be incomplete pericardial resection with residual posterolateral or diaphragmatic pericardium. ${ }^{(54)}$ The local report from 1982, and the follow up anatomically based study, would therefore suggest that a left anterolateral approach would be the most suitable approach for undertaking a radical pericardiectomy in a population with a high prevalence of TBP. In our local experience a limited anterior pericardial resection, phrenic nerve to phrenic nerve, is most commonly employed via median sternotomy for procedural safety reasons. The decision to extend this to include the posterolateral and diaphragmatic pericardial surfaces is assessed on a case-by-case basis, weighing up the risk of intraoperative haemorrhage against that of the potential for recurrence of CP. Whether or not pericardiectomy via median sternotomy is associated with recurrence of $\mathrm{CP}$ in our population is currently not known. 


\section{CONCLUSION}

TBP continues to cause unacceptably high rates of morbidity and mortality despite more than 6 decades of antituberculous medical therapy directed against it. Regardless of modern advances in medicine, this is clearly not a disease for which there will be a suitable solution in the near future. It remains a diagnostic challenge and the development of a point of care test, stringently evaluated against confirmed TBP by demonstration of the bacillus in fluid or tissue specimens, would be of immense value to the diagnosis and management of the disease in SSA. The potential value of intrapericardial antituberculous chemotherapy should be explored as a possible means of impacting the time to cure and reducing the associated morbidity and mortality. In 2012 the worldwide incidence of MDR TB stood at 450000 whilst the rates of drug resistant TBP still remain undefined. Bedaqualine, delaminid and pretomanid, drugs in development for resistant TB, are highly protein-bound which may influence their availability within the pericardial space and their potential value in the treatment of resistant pericardial disease will have to be assessed. In HIV negative patients who present with tuberculous ECP, the potential value of adjunctive steroids should be evaluated against those who present with pure effusive disease and undergo complete pericardial drainage. It is highly probable that those in the effusive-constrictive phase stand to benefit most from immunomodulation of the host response. A detailed TTE by an experienced echocardiographer, employing TDI and hepatic venous PW-Doppler, can confidently diagnose constrictive pericarditis and differentiate it from restrictive cardiomyopathy. The application of the recently proposed Mayo Clinic TTE criteria for the diagnosis of CP should be used to establish, or refute, a possible diagnosis of tuberculous CP. The applicability of these criteria should also be evaluated in establishing a diagnosis of recurrent $\mathrm{CP}$ in a population with a high prevalence of TBP. In those with constriction, a left anterolateral thoracotomy would appear to offer a more suitable approach for achieving successful radical pericardiectomy vs. that via median sternotomy. Most surgical centers in SA undertake pericardiectomy via a median sternotomy and the rate of recurrent constriction in this group needs to be formally evaluated against that of an approach via left anterolateral thoracotomy in the HIV era. TBP remains a challenging disease, to both diagnose and manage, and it should be an area of research priority within our academic cardiology and cardiothoracic surgery units in SSA. It is predominantly a disease of the African continent that requires a solution from within this region.

\section{Conflict of interest: none declared.}

\section{REFERENCES}

I. WHO, Global tuberculosis report 2014. Geneva: World Health Organisation; 2014 (http://www.who.int/tb/publications/global_report/en).

2. Hershkovitz I, Donoghue HD, Minnikin DE, et al. Detection and molecular characterisation of 9000-year-old Mycobacterium tuberculosis from a Neolithic settlement in the Eastern Mediterranean. PLOS ONE 2008;3:e3426.

3. East African/British Medical Research Councils: Controlled clinical trial of 4 short course (6-month) regimens of chemotherapy for treatment of pulmonary tuberculosis. Second report. Second East African/British Medical Research Council Study. Am Rev Respir Dis 1976; | | 4:47 I -475.

4. WHO, Global tuberculosis report 20 I3. Geneva: World Health Organisation 2013 (http://www.who.int/tb/publications/global_report/en).

5. Caws $M$, Thwaites $G$, Dunstan $S$, et al. The influence of host and bacterial genotype on the development of disseminated disease with Mycobacterium tuberculosis. PLoS Pathog 2008;4(3):el 000034.

6. Larrieu AJ, Tyers GF, Williams EH, et al. Recent experience with tuberculous pericarditis. Ann Thorac Surg 1980;29(5):464.

7. Reuter $\mathrm{H}$, Burgess LJ, Doubell AF. Epidemiology of pericardial effusion in a large academic hospital in South Africa. Epidemiol Infect 2005; 133:393-399.

8. Mayosi BM, Wiysonge CS, Ntsekhe M, et al. Mortality in patients treated for tuberculous pericarditis in sub-Saharan Africa. S Afr Med J 2008;98:36-40.

9. Reuter H, Burgess LJ, Louw VJ, Doubell AF. The management of tuberculous pericardial effusion: Experience in 233 consecutive patients. Cardiovasc J S Afr 2007; 1 8:20-25.

10. Schrire V. Experience with pericarditis at Groote Schuur Hospital, Cape Town: Analysis of 160 cases studied over a 6-year period. S Afr Med J 1959;33:810-7.

II. Reuter H, Burgess LJ, Louw VJ, Doubell AF. Experience with adjunctive corticosteroids in managing tuberculous pericarditis. Cardiovasc J S Afr 2006;5:233-238.

12. Cui HB, Chen $X Y$, Cui CC, et al. Prevention of pericardial constriction by transcatheter intrapericardial fibrinolysis with urokinase. Chin Med Sci J 2005;20:5-10.

13. Mayosi BM, Ntsekhe M, Bosch J, et al. Prednisolone and Mycobacterium

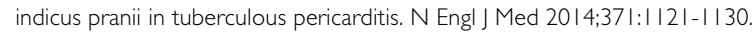

14. Heemskerk AD, Bang ND, Mai NTH, et al. Intensified antituberculosis therapy in adults with tuberculous meningitis. N Engl J Med 2016:374: 124-34.

15. Mayosi BM, Ntsekhe M, Volmink JA, et al. Interventions for treating tuberculous pericarditis. Cochrane Database Syst Rev 2002;4:CD000526.

16. Treatment Action Group (TAG), Report on tuberculosis research funding trends, 2005-2014: A decade of data 2015. (http://www.treatmentaction group.org/tbrd20 I5).

17. Diacon AH, Pym A, Grobusch MP, et al. Multidrug-resistant tuberculosis and culture conversion with bedaquiline. N Engl J Med 20 |4;371:723-32.

18. Reuter H, Burgess LJ, van Vuuren W, Doubell AF. Diagnosing tuberculous pericarditis. QJM 2006;99:827-839.

19. Mayosi BM, Wiysonge CS, Ntsekhe M, et al. Mortality in patients treated for tuberculous pericarditis in sub-Saharan Africa. S Afr Med J 2008;98:36-40.

20. Burgess LJ, Reuter H, Carstens ME, et al. The use of adenosine deaminase and interferon- $\gamma$ as diagnostic tools for tuberculous pericarditis. Chest 2002; 1 22:900-905.

21. Friedrich S, von Groote-Bidlingmaier, Diacon A. Xpert MTB/RIF assay for the diagnosis of pleural tuberculosis. J Clincl Microbiol 20 I 1;49( I 2):434 I-4342.

22. Pandie S, Peter JG, Kerbelker ZS, et al. Diagnostic accuracy of quantitative PCR (Xpert MTB/RIF) for tuberculous pericarditis compared to adenosine deaminase and unstimulated interferon- $\gamma$ in a high burden setting: $A$ prospective study. BMC Med 2014;12:101-111.

23. Cegielski J, Devlin B, Morris A, et al. Comparison of PCR, culture and histopathology for the diagnosis of tuberculous pericarditis. J Clincl Microbiol 1997;35:3254-3257.

24. Seferovic PM, Risitc AD, Maksimovic R, et al. Diagnostic value of pericardial biopsy: Improvement with extensive sampling enabled by pericardioscopy. Circulation 2003; 107(7):978-983. 
25. Maisch B, Drude L. Pericardioscopy-a new diagnostic tool in inflammatory diseases of the pericardium. European Heart Journal 1991; 2:Suppl D:2-6.

26. Maisch B, Rupp H, Ristic A. Pericardioscopy and epi- and pericardial biopsy-a new window to the heart improving etiological diagnoses and permitting targeted intrapericardial therapy. Heart Failure Review 2013. Online publication 12 March 2013.

27. Seferovic PM, Ristic AD, Maksimovic R. Flexible percutaneous pericardioscopy: Inherent drawbacks and recent advances. Herz 2000;25(8): $741-747$.

28. Nugue $\mathrm{O}$, Millaire A, Porte $\mathrm{H}$, et al. Pericardioscopy in the etiologic diagnosis of pericardial effusion in 141 consecutive patients. Circulation 1996; 94: 1635-164|.

29. Maisch B, Pankuweit S, Brilla C, et al. Intrapericardial treatment of inflammatory and neoplastic pericarditis guided by pericardioscopy and epicardial biopsy - results from a pilot study. Clin Cardiol 1999;22(Suppl I): 117-22.

30. Kyriakakis CG, Weich HS, Doubell AF. Percutaneous pericardioscopy in tuberculous pericarditis: Improving the diagnostic yield. European Heart Jnl 201 1;32(Suppl):487

31. Zumla A, Raviglione M, Hafner R, et al. Tuberculosis. New Engl J Med 2013:368:745-55

32. Hesseling A, Marais B, Kirchner H, et al. Mycobacterial genotype is associated with disease phenotype in children. Int J Tuberc Lung Dis 2010; 14(10): 1252-1258.

33. Garcia de Viedema D, Lorenzo G, Cardona PJ. Association between the infectivity of Mycobacterium tuberculosis strains and their efficiency for extrapulmonary infection. J Infect Dis 2005; 192:2059-2065.

34. Gandhi N, Brust J, Moodley P, et al. Minimal diversity of drug-resistant Mycobacterium tuberculosis strains, South Africa. Emerging Inf Dis Jnl 2014:20(3):426-433.

35. Ntsekhe M, Wiysonge C, Volmink JA, et al. Adjuvant corticosteroids for tuberculous pericarditis: Promising, but not proven. QJM 2003;96(8):593-9.

36. Adler Y, Charron P, Imazio M, et al. 2015 ESC Guidelines for the diagnosis and management of pericardial diseases. The task force for the diagnosis and management of pericardial diseases of the European Society of Cardiology (ESC). Eur Heart Jnl 2015;36:2921-2964.

37. Treatment of tuberculosis. American Thoracic Society, CDC, Infectious Diseases Society of America. MMWR Recomm Rep 2003;52(RR-I I): I-77.

38. Supplement to: Mayosi BM, Ntsekhe M, Bosch J, et al. Prednisolone and Mycobacterium indicus pranni in tuberculous pericarditis. N Engl J Med 2014;37|: | | 21-30. DOI: 10.1056/NEJMoa 407380.

39. Garcia MJ, Rodriguez L, Ares M, et al. Differentiation of constrictive pericarditis from restrictive cardiomyopathy: Assessment of left ventricular diastolic velocities in longitudinal axis by Doppler. JACC 1996;27(1): 108-14.

40. Ha JW, Oh JK, Nishimura RA, et al. Annulus paradoxus: Transmitral flow velocity to mitral annular velocity ratio is inversely proportional to pulmonary capillary wedge pressure in patients with constrictive pericarditis. Circulation 200I; 104:976-978.

41. Welch TD, Ling LH, Espinosa R, et al. Echocardiographic diagnosis of constrictive pericarditis: Mayo Clinic criteria. Circ Cardiovasc Imaging 20 I4; 7:526-534.

42. Rajagopalan N, Garcia MJ, Rodriguez L, et al. Comparison of new Doppler echocardiographic methods to differentiate constrictive pericardial heart disease from restrictive cardiomyopathy. Am J Cardiol 2001;87:86-94.

43. Sagristà-Sauleda J, Angel J, Sanchez A, et al. Effusive-constrictive pericarditis. N Engl J Med 2004;350:469-475.

44. Mayosi BM, Burgess LJ, Doubell AF. Tuberculous pericarditis. Circulation 2005; | 12:3608-36 |6

45. Imazio M, Brucato A, Maestroni S, et al. Risk of constrictive pericarditis after acute pericarditis. Circulation 20 I 1; 124( I I): 1 270-1275.
46. Long $\mathrm{R}$, Younes $\mathrm{M}$, Patton $\mathrm{N}$, et al. Tuberculous pericarditis: long-term outcome in patients who received medical therapy alone. Am Heart J 1989; $117(5): 1133$

47. Chowdhury UK, Subramaniam GK, Kumar AS, et al. Pericardiectomy for constrictive pericarditis: A clinical, echocardiographic and haemodynamic evaluation of two surgical techniques. Ann Thorac Surg 2006;81:522-529.

48. Pitt Fennel WM. Surgical treatment of constrictive tuberculous pericarditis. South Afr Med J 1982;62:353-355.

49. Lachman N, Vanker EA, Christensen BA, et al. Pericardiectomy: A functional anatomical perspective for the choice of left anterolateral thoracotomy. J Card Surg 2009;24:41 |-413.

50. Mutyaba AK, Balkaran $\mathrm{S}$, Cloete $\mathrm{R}$, et al. Constrictive pericarditis requiring pericardiectomy at Groote Schuur hospital, Cape Town, South Africa: causes and perioperative outcomes in the HIV era (1990-2012). J Thoracic Cardiovasc Surg 20|4; 148:3058-3065.

5I. Szabo G, Schmack B, Bulut C, et al. Constrictive pericarditis: risks, aetiologies and outcomes after total pericardiectomy: 24 years of experience. Eur J Cardiothorac Surg 2013;44:1 023-1028.

52. Ha JW, Oh JK, Schaff HV, et al. Impact of left ventricular function on immediate and long-term outcomes after pericardiectomy in constrictive pericarditis. J Thorac Cardiovasc Surg 2008; | 36(5): | |36-| | 4 |.

53. Biçer M, Özdemir IK, Yüksel A, et al. Long-term outcomes of pericardiectomy for constrictive pericarditis. Jnl of Cardiothorac Surg 20 I5; 10:177-181.

54. Cho YH, et al. Completion pericardiectomy for recurrent constrictive pericarditis: Importance of timing of recurrence on late clinical outcome of operation. Ann Thoac Surg 2012;93:1236-1240. 